\title{
System Engineering in the Energy and Maritime Sectors: Towards a Solution Based on Model-Centric Processes
}

\author{
Lionel Briand \\ Simula Research Laboratory \\ University of Oslo
}

\begin{abstract}
The Maritime and Energy industry is facing rapid change with an increasing reliance on software embedded systems and integrated control and monitoring systems. From a practical stand point, challenges are related to increased system complexity, increasingly integrated sub-systems relying on Commercial-Of-The-Shelf software, longer supply chains for equipment and components delivered by different suppliers, and short duration for construction and commissioning of ships and offshore platforms. As a result, there is a lack of visibility into the architecture of systems, their design rationale, how subsystems/components were verified and integrated, and finally how systems were validated and certified with a particular focus on safety. In turn, this has hindered effective collaboration among stakeholders, including suppliers and system integrators.

This talk will present a recent initiative, led by Simula Research Laboratory and Det Norske Veritas (DNV), Norway, to address the above problems. The general approach relies on model-centric processes, where models of the system specifications, architecture, and design properties, are used to support the documentation of architecture and design rationale, traceability among development artifacts, and guide safety analysis and testing, among other things. The project is focused on devising novel but scalable approaches to the longstanding model-driven development challenges.
\end{abstract}

